\title{
A FUNDAMENTAL IDEA OF UNDERSTANDING A SENTENCE GRAMATICALLY
}

\author{
Nizamuddin Sadiq \\ Islamic University of Indonesia
}

\begin{abstract}
To study of syntax, the concept of structure is fundamental. However structure itself is a very general concept and most of the language leamers are assumed that their understanding about sentence is obvious. For many people, a sentence is considered group of words. This idea is not appropriate when a deep study of fundamental idea of a sentence is done.

Linguistically speaking, sentences are not formed by words alone but by structural units known as constituents. The constituent has a certain specifiable function in the structure of the thing as a whole. However, the. fundamental idea of a sentence grammatically is that a sentence consists of phrase. Therefore, sentence (S) consists of noun phrase (NP) and verb phrase (VP). The idea of phrase here is to accommodate even a single constituent that a sentence has. The basic principle of sentence, therefore, is $S=N P+V P$. It is obvious that NP and VP directly form a sentence. Furthermore, in terms of relation that the phrase may have in a sentence, there are four kinds of phrase relation in a sentence. They are modification, complementation, predication, and coordination.
\end{abstract}

Keywords: syntax, constituent, words, phrase, NP, and VP

\section{A. Introduction}

Most of leamers will deal with a sentence when learning a language. As we know that, a sentence is the largest grammatical unit in any language. However, when it comes to English, everybody will be assumed that they already have an intuitive idea of what counts as a sentence of English. Therefore, the question, 'What do sentences consist of?' is the basic thing to understand. The answer might seem blindingly obvious: Sentences consist of words. This apparently natural answer is not the most appropriate one.

Linguistically speaking, sentences are not formed by words alone but by structural units known as constituents. Nevertheless, an understanding of how the 
constituent parts of a sentence functions, in order to fully understand how sentences are formed and organized is essential. This paper is trying to describe the fundamental idea of grammatically what a sentence is.

\section{B. Discussion}

To understand the idea of what a sentence grammatically is, describing what English sentence is will be great help. Therefore, to begin with, a fundamental distinction between two kinds of sentences: Kernel and Transformation sentence must be made clear first. According to Robert (1964), Kernel sentences are the basic, elementary sentences of the language, the stuff from which all else is made, while Transformation sentences are all else structure down from the KerneI sentences to provide all the complication of English sentences.

Lyons (1968) defined Kernel sentences as any sentence, which is, generated from a single kernel string without the application of any optional transformation. Transformation, according to Dinneen (1967) is addition, deletion or change in order to or form of the morphemes involved, which enables us to relate many utterances that appear on the surface, to be simply different.

It is obvious, then, Kernel sentences are a kind of basic sentences and transformations are a kind of sentences with any change. Because Kernel sentences are the basic, it is surely that we shall see its basic rule.

Robert (1964) states that all Kernel sentences contain tow main parts: a noun phrase (NP) and a verb phrase (VP). The NP functions as the subject and the VP as its predicate. The NP and the VP are used to include single words as well as groups of words. It is simply that the term phrase to mean sometimes groups of words and sometimes-single words. Another expert supported the Robert's definition. Kolln said that a complete sentence is containing a noun phrase subject and a verb phrase predicate. A noun phrase (which can be as short as one word) is the subject, whereas the verb phrase is the rest of the sentence-containing verb. The verb phrase can be as short as one word.

From what some experts said above, I do agree to say that grammatically a sentence does not consist of words but phrases. By using abbreviation, it might say that a sentence consist of NP+VP. Thus, sentence (S) consists $\rightarrow$ NP+VP.

$$
\mathrm{S}=\mathrm{NP}+\mathrm{VP}
$$

Table 1. Sentence units 
The examples will be given in the following.

1. a. David landed the plane. The NP that functions as subject is David.

b. The pilot landed the plane. The NP that functions as subject is the Pilot.

2. a. The plane arrived. The VP that functions as predicate is arrived.

b. The pilot landed the plane. The VP that functions as predicate is landed the plane.

Therefore, David and Pilot are considered as noun phrase and arrived and landed the plane are considered as verb phrase. It is very obvious then a sentence consists of NP + VP. It means that NP + VP directly form a sentence. So far, the focus is on simple sentence with a basic subject + predicate construction; however, to add information, it sometimes needs an additional element in the sentence. This is often a type of adverbial phrase.

\section{Why optional?}

As Kolln explains, the term adverbial refers to any grammatical structures that answer the questions:

When : When Michael wakes up, he eats breakfast.

Where: My dog always races to the dog park.

Why :To improve my Spanish, I will spend the summer in Belize.

Annie plays basketball because she loves teamwork and competition.

How : He solved the problem with great difficulty.

Although adverbial may be necessary for content, they are considered optional because they can be eliminated, and the remaining words will still form a grammatical sentence. For example,

Annie plays basketball because she loves teamwork and competition.

\section{Adverbial}

When the adverbial is removed, Annie plays basketball still contains the required elements of a sentence: a subject and a predicate. In addition, the placement of adverbial is often optional, depending on the adverbial's grammatical structure. The sentence about Annie has three optional slots: 
1. Before the subject.

Because she loves teamwork and competition, Annie plays basketball.

(Notice that a comma is needed after the adverbial since it comes before the subject.

2. Between the subject and predicate.

Annie, because she loves teamwork and competition, plays basketball.

(Notice that commas are needed AROUND the adverbial since it comes between the subject and the verb).

3. At the end of the sentence.

Annie plays basketball because she loves teamwork and competition.

(No comma is needed when the adverbial comes at the end of the sentence).

\section{Types of Adverbials}

Furthermore, Kolln discuses four types of grammatical structures for adverbial. Thy will be reviewed here. Adverbs are single words that modify (describe) verbs, adjectives, other adverbs, and complete sentences:

A verb:

Brittany

(sang)

Verb

beautifully.

An adjective:

Her song was

absolutely

Adverb

Another adverb: Brittany sang

A sentence: very

Adverb

Cerfainly,

Adverb adverb

(lovely.)

adjective

(beautifully.)

adverb

(Britlany sang well.)

sentence

Prepositional phrases are made up of a preposition plus a noun phrase:
On the beach
$\mathrm{P} \mathrm{NP}$
In March
After the game
P
For mvmom
P NP 
Prepositions are usually used to give information about direction, location, purpose, or time, and they can be used in either the subject or the predicate.

\begin{tabular}{llll}
\hline \multicolumn{2}{c}{ Subject } & & Predicate \\
\hline We & found my sweatshirt & (on the beach.) \\
& & & P + NP = PP \\
& & (Preposition Phrase) \\
\hline That present & (for my mom) & Was & expensive. \\
& $\mathrm{P}+\mathrm{NP}=\mathrm{PP}$ & & \\
\hline
\end{tabular}

Table 2. Preposition

Subordinate clauses (SC) are made up of a subordinator + subject + predicate: Annie plays basketball because she lovesteamwork and competitive. Subordinator + subject + verbphrase (predicate) $=\mathrm{SC}$ Infinitives are made up of to + verb phrase:

To improve mv Spanish, I will spend the summer in Belize.

To + verb phrase $=$ infinitive

That is to say, the phrases, which indirectly form sentences, are adverbial in the terms of those adverbials are single words that modify (describe) verbs, adjectives, other verbs, and complete sentence. Prepositional phrases are made up of a preposition plus a noun phrase. Subordinate clauses are made up of a subordinator + subject + predicate. Infinitives are made up of to + verb phrase.

\section{Phrase Elements}

Linguistically speaking, the elements of phrases are head, complement, attribute, adjunct, and specifier. Head is a constituent whose distribution is the same as that the reluctant construction. Complement is an element, which a head semantically requires to complete its meaning (gives/implies action and directly related/connected). Attribute is an element of a phrase, which shows additional information (characteristic) of a head and comes before the head. Adjunct is an element of phrase, which shows additional information (characteristic) of a head 
and comes before the head. Specifier indicates how many such objects are being described, as well as how the objects being described relate to the speaker and the hearer. Specifier is constructed out of ordinals, cardinals, and determiners. For example,
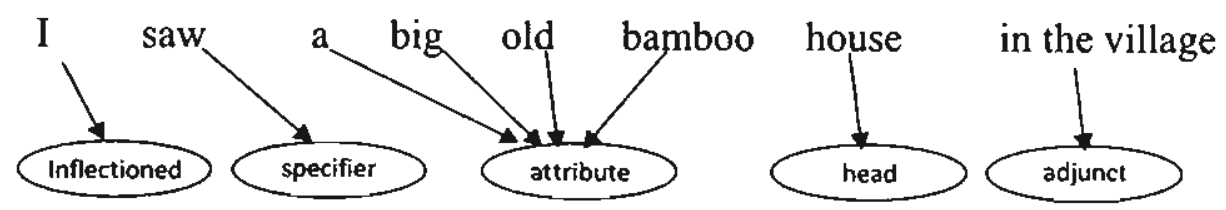

Those elements above can be drawn into the level in the following way:

\begin{tabular}{|c|}
\hline I \\
N \\
$T$ \\
$E$ \\
$R$ \\
$M$ \\
$E$ \\
$D$ \\
I \\
$A$ \\
$T$ \\
$E$ \\
L \\
E \\
$V$ \\
$E$ \\
L \\
\hline
\end{tabular}

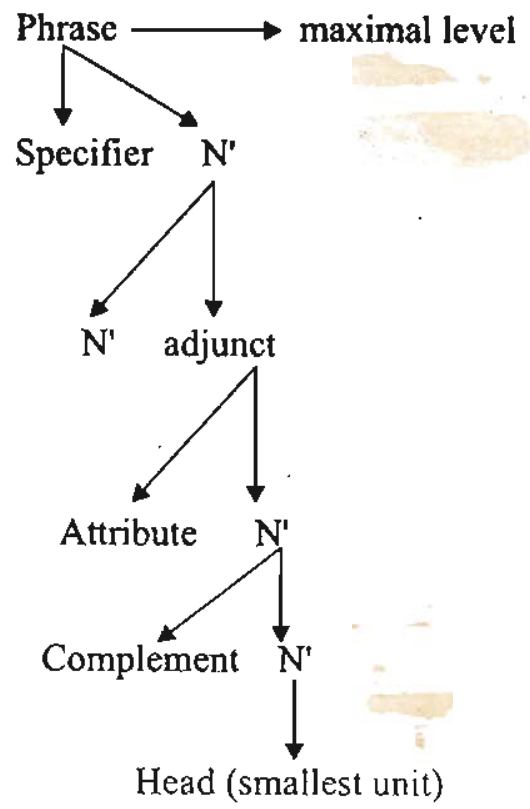

By considering those elements above, there will be four kinds of phrase relation in a sentence.

a. Modification (modified modifier) $(\rightarrow$ )

b. Complementation (verb object/predicate object((C)

c. Predication ( subject predicate)(P)

d. Coordination (two sentences or clauses $(=)$ 
Examples:

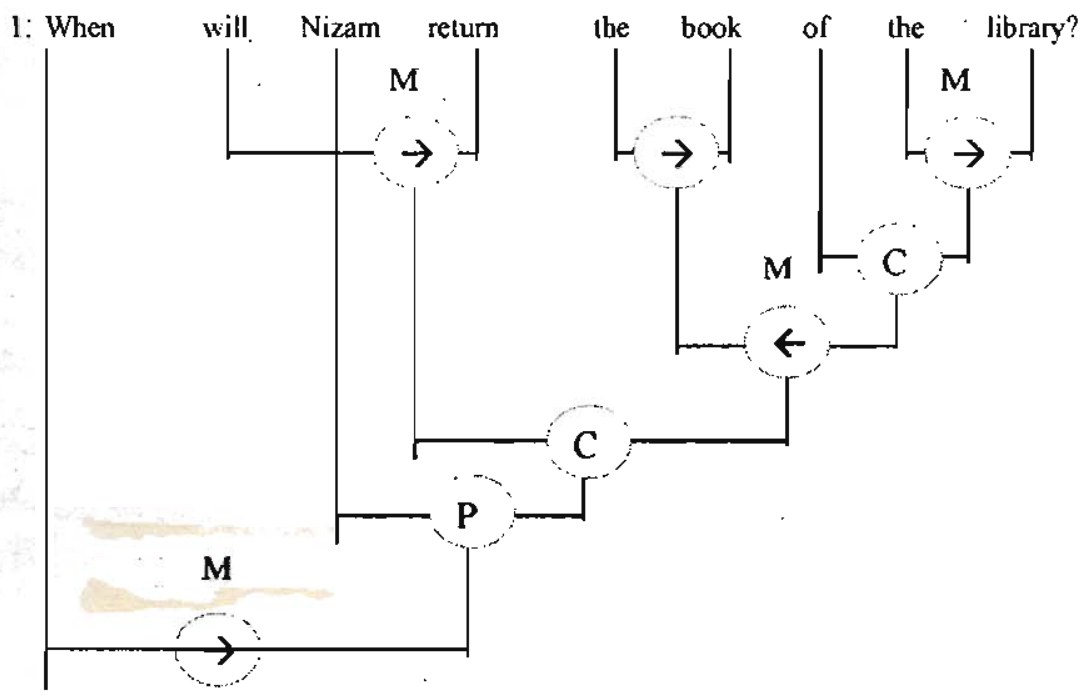

2.

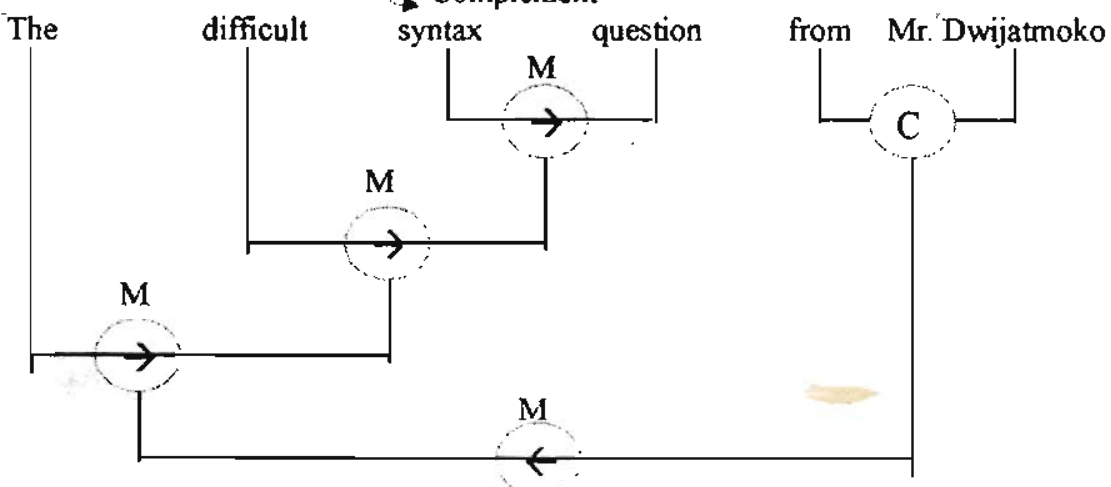




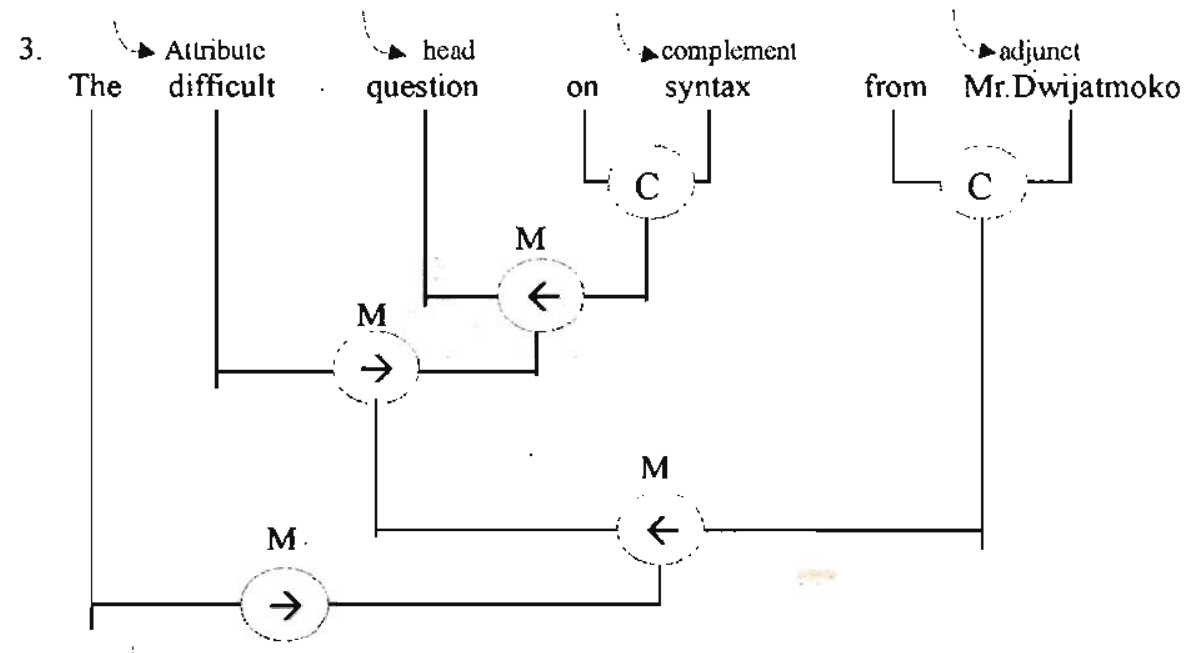

4.

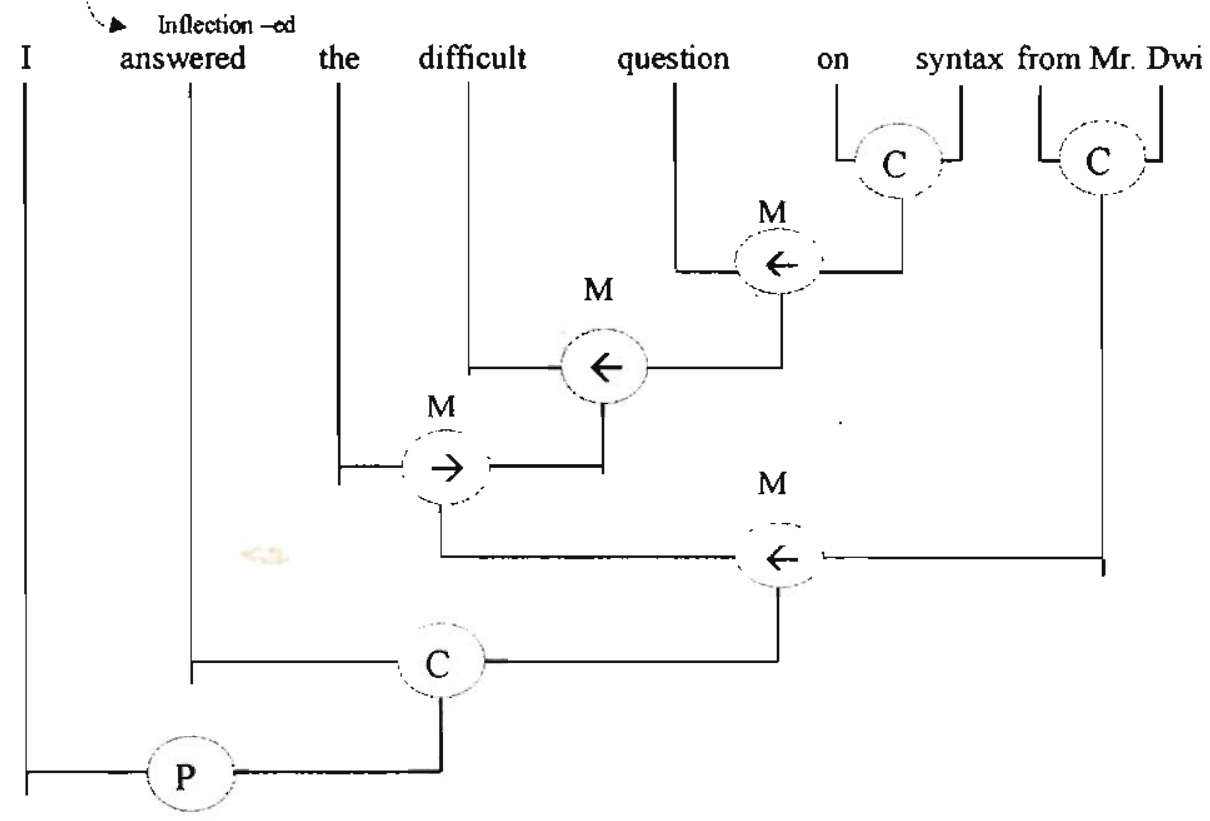




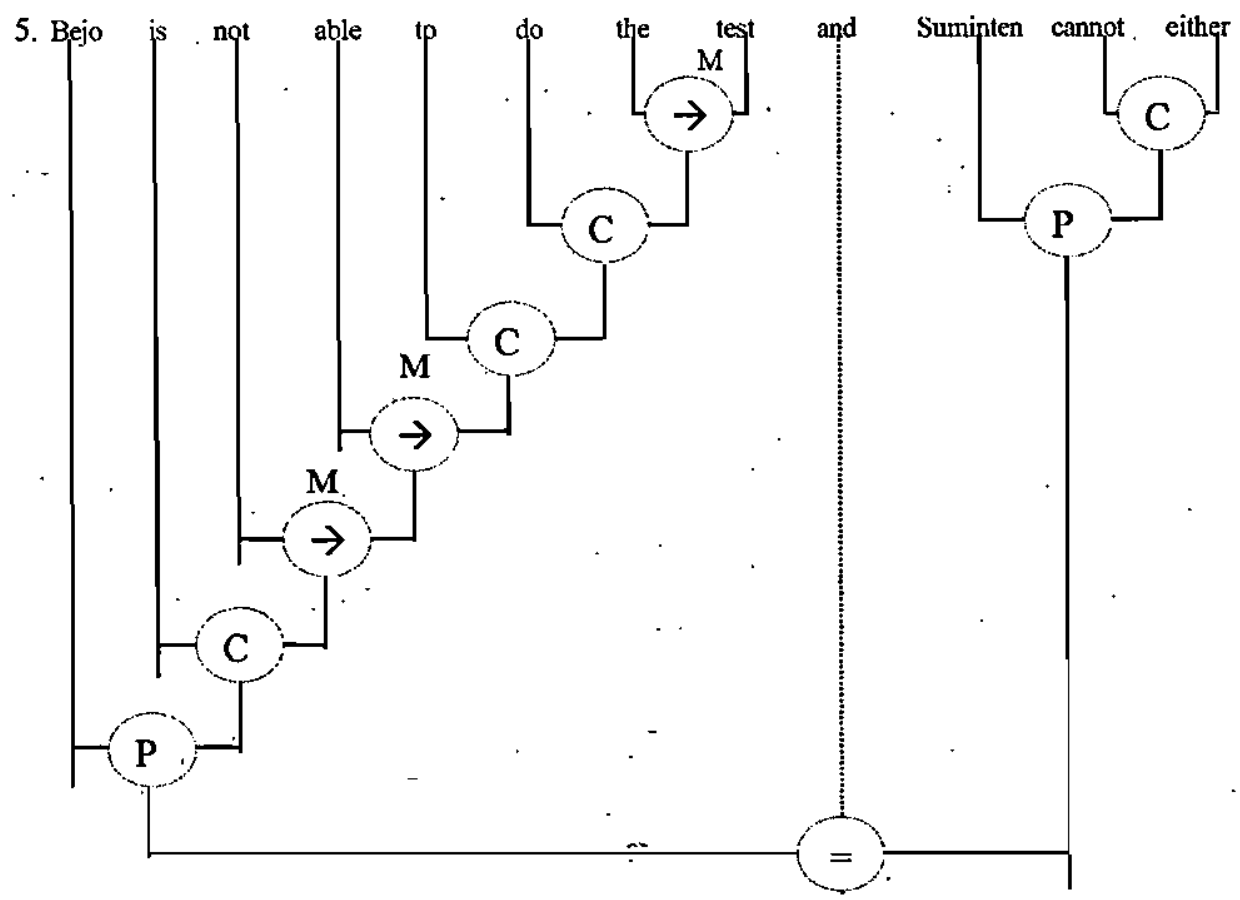

In different ways Bubba proposed the relation of phrase that may have in sentence by describing unit that appears in sentences and its function. Bubba stated that it is very important in syntax to distinguish between what kinds of units act as building blocks in syntax, on the one hand, and the particular roles these building blocks play in larger syntactic construction. He proposed the table to set out three kinds of information: the linguistic terms for the units that appear in sentences (word, phrase, clause, and sentence); the linguistic terms for different classes or categories of those units (a noun $(=N)$ ) is a kind of word, a NP (noun phrase) is a kind of phrase. The third column lists the finctions that these different units play in building sentences; these functions are based on meaning relationship among the units, and uses that the units are put to in communication.

The units of syntactic structure (building blocks of phrases, clauses, and sentences) will be displayed in the table 3 . 


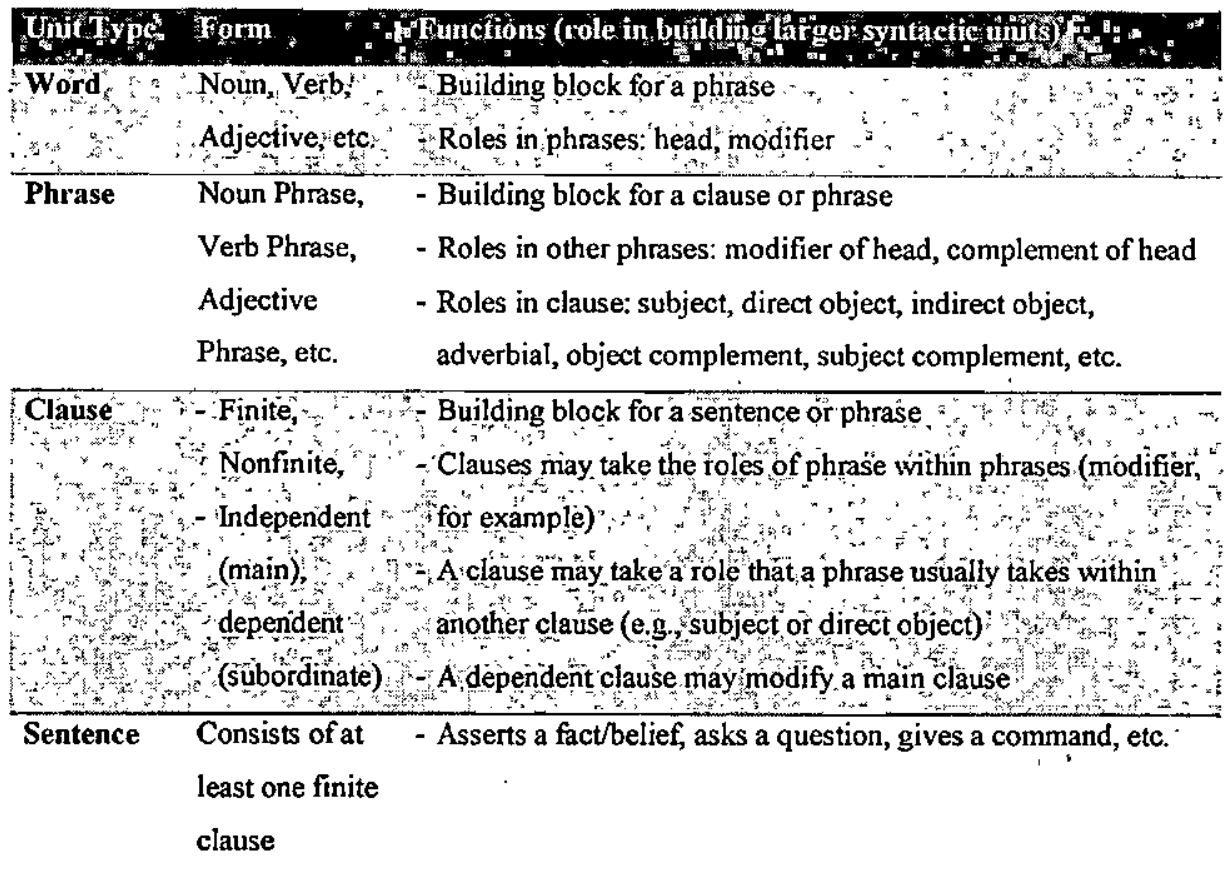

Table 3. The units of syntactic structure

Those units will then be described in the following examples.

A. Roles in other phrases

1. Modifier head

a. My fuzzy black poodle barks too much.

Fuzzy ${ }^{-}=$Modifier

Black $=$ Modifier

Poodle = Noun Head

b. My fuzzy black poodle barks too much.

Barks $\quad=$ Head verb

Toomuch $=$ Modifier

2. Complement of head

a. The president of the company.

The president $=$ Head

Of the company $=$ Complement 
B. Roles in clauses

b. His desire to succeed

His desire $\quad=$ Head

Tosucceed $=$ Complement

1. As subject

a. Justin kicked the ball.

Justin $\quad=$ Noun Phrase/ subject.

b. The present for my mother was expensive.

For my mom $=$ Preposition phrase $/$ subject.

2. As direct object

a. Tom gave the book to Marni.

The book $=$. Noun Phrase/direct object

b. That sweater on the floor has grass strain on the sleeve.

Agrass strain $=$ Noun Phrase/direct object.

3. As indirect object

a. The firm has offered Tania a job.

Tania = Noun Phrase/indirect object

b. My grandmother called my son a cute boy.

My son . = Noun Phrase/indirect object.

4. As Adverbial

a. Tanty sings well.

Well = Adverb Phrase/adverb

b. Wenger lives in London.

In London $=$ Preposition Phrase/adverb.

5. As Object complement

a. The firm has offered Melati a job.

Ajob $=$ object complement.

b. My grandmother called my son a cute boy.

Acute boy $=$ object complement

6. As subject complement

a. Ariella is a writer.

Awriter = subject complement

b. Ms. Lely is a diligent secretary.

Adiligent secretary . = subject complement 


\section{Conclusion}

Although it seems understandable that a sentence consists of words, the fundamental concept of a sentence grammatically has been revealed otherwise. A sentence do contains of phrase. The idea of phrase does not mean phrase of natural facts that they have at least two visible words chained together. Conversely, a single syntactic unit can be considered a phrase because the fundamental idea of phrase is that NP ? (Det) (Adj) N. When N is occurs alone, N belongs to NP. In creating sentence, every part of units has their own function. There are four kinds of phrase relation in a sentence that are modification, complementation, predication, and coordination: ${ }^{\cdots-}$

\section{References}

Dinneen, Francis.P. 1967. An Introduction to General Linguistics. Georgetown University Press: Washington DC.

Lyons, John. 1968. Introduction to Theoretical Linguistics. Cambridge University Press: Cambridge. ‘.

Robert, Paul. 1964. English Syntax. Alternate Edition. Harcourt Brace World Inc: New York.

http://www.cla.calpoly.edu/ jrubba/syn/SyntaxT\&C.html 